\title{
Promotion of influenza vaccination among health care workers: findings from a tertiary care children's hospital in Italy
}

Vanessa Cozza ${ }^{1,2}$, Valeria Alfonsi ${ }^{3}$, Maria Cristina Rota ${ }^{3}$, Valerio Paolini ${ }^{2}$ and Marta Luisa Ciofi degli Atti ${ }^{4^{*}}$

\begin{abstract}
Background: The aims of this study were: a) to evaluate attitudes and practices of health care workers (HCWs) towards influenza vaccination and their opinion regarding a vaccination promotion toolkit; b) to estimate hospital HCWs' influenza vaccination coverage rates (VC).

Methods: The Bambino Gesù Children's Hospital (OPBG) is an academic hospital in Italy. Since 2009, free influenza vaccination is offered to HCWs during working hours. In October-December 2013, a communication campaign based on a standardized toolkit was conducted. In December 2013, we performed a cross-sectional survey in a sample of hospital wards, based on a self-administered questionnaire including participants' characteristics; self-reported influenza vaccination history; reasons for vaccination or missed vaccination; opinion regarding the toolkit. Multivariable logistic analysis was used to assess independent predictors of influenza vaccination status. Annual VC for years 2009-2013 was estimated by using the number of seasonal influenza vaccine doses administered to HCWs as numerator, and the number of hospital HCWs as denominator.
\end{abstract}

Results: Out of 191 HCWs who participated in the survey, $35.6 \%$ reported at least one influenza vaccination during their life; $6.8 \%$ adhered to annual revaccination. Years of service and professional category were significantly and independently associated with vaccination (adjusted-OR: 2.4 for $>10$ years of service, compared to $<5$ years of service; adjusted-OR: 2.6 for physicians compared to nurses). Patient protection was the main reported reason for vaccination (34.3\%); considering influenza a mild disease was the main reason for non-vaccination (36.9\%); poor vaccine effectiveness was the main reason for missed annual revaccination (28.8\%). Overall, $75 \%$ of respondents saw at least one promotion tool; $65.6 \%$ of them found the information useful. Hospital VC decreased from $30 \%$ in 2009, to $5 \%$ in 2012. In 2013, VC was $14 \%$.

Conclusions: Satisfactory influenza VC in HCWs is hard to achieve. In 2013, along with the toolkit implementation, we observed an increase in HCWs' vaccination coverage, nevertheless, it remained unsatisfactory. Tailored information strategies targeting nurses and recently employed HCWs should be implemented. Institution of declination statements, adding influenza vaccination to financial incentive systems, or vaccination requirements should also be considered to increase influenza VC among HCWs.

Keywords: Influenza vaccine, Healthcare workers, Communication campaign, Attitudes

\footnotetext{
* Correspondence: marta.ciofidegliatti@opbg.net

${ }^{4}$ Clinical Epidemiology Unit, Medical Direction, Bambino Gesù Children's

Hospital, Piazza Sant'Onofrio, 4, Rome 00165, Italy

Full list of author information is available at the end of the article
} 


\section{Background}

Vaccination against seasonal influenza is recommended to health care workers (HCWs) by national and international institutions [1-4], in order to reduce the risk of acquiring influenza and transmitting the infection to vulnerable patients [5-8]. Influenza outbreaks may also cause HCW' absenteeism, resulting in inadequate staffing, diminished quality of care and increased costs $[9,10]$.

A recent meta-analysis confirmed that HCWs' influenza vaccination is effective in preventing mortality and influenza cases among patients of healthcare facilities [11]. However, influenza vaccination coverage among HCWs remains suboptimal worldwide [12-15]. In Europe, data from 10 countries for the 2010-2011 influenza season showed a vaccination coverage $<35 \%$ in 8 countries, and ranging from 41 to $64 \%$ in the remaining two countries [14].

In Italy, influenza vaccination of $\mathrm{HCWs}$ is recommended by the Ministry of Health and is offered free of charge by the national health service (NHS) [4]. However, data on vaccination coverage (VC) among HCWs are not routinely collected and the few ad-hoc studies have consistently found low coverage rates (12-34 \%), also during the 2009-2010 pandemic [16-18]. Misperceptions about the severity of influenza, lack of knowledge on the benefits of the vaccination and fear of adverse events are frequently reported as reasons for missed vaccination $[12,16,18]$. Perceived lack of leadership support is also a potential barrier to HCWs' influenza vaccination [12].

In 2011, the European Commission funded the HProImmune project [19], aiming at increasing awareness among HCWs of several vaccine preventable diseases, enhancing their knowledge on immunization and promoting vaccinations. The HProImmune consortium comprised 10 associated partners from 7 countries (Greece, Romania, Poland, Lithuania, Italy, Cyprus and Germany) and 2 European collaborating partners (WHO/EURO Centre for Environment and Health and Health Protection Agency - UK).

Within this project, influenza vaccination was identified as a priority and a toolkit for immunization promotion was developed on the basis of healthcare personnel's needs and perspectives, as identified through a literature review and through qualitative methods (i.e. focus groups) exploring behaviours and barriers towards immunization. In Autumn 2013, the Bambino Gesù Children's Hospital, the largest Italian children's hospital, used the HProImmune influenza toolkit to promote influenza vaccination for HCWs.

In this article, we present: a) the results of a survey conducted among HCWs to assess attitudes and practices regarding influenza vaccination, and opinion regarding the toolkit; b) estimates of the hospital's HCWs' vaccination coverage rates for influenza from 2009 to 2013.

\section{Methods}

Vaccination offer and communication campaign

The Bambino Gesù Children's Hospital (OPBG) is a tertiary care academic hospital, with 607 inpatient-bed, located in Rome, Italy. Since 2009, free influenza vaccination is offered to HCWs through mobile teams and dedicated vaccination sessions, from October to December. Information about the vaccination offer is sent to all hospital wards and posted on the hospital's intranet. The number of influenza vaccine doses administered to HCWs is recorded by the Medical direction. In OctoberDecember 2013, a communication campaign based on the HproImmune influenza toolkit was conducted. The communication tools consisted in: i) posters, placed in areas which were frequently visited by HCWs (changing rooms, attendance recorder area, canteen, etc.); ii) information factsheets, distributed in paper form in hospital wards and also available in electronic format on the hospital's intranet; iii) a banner on the hospital's intranet, linked to the electronic information factsheets. The campaign's key messages focused on personal protection and patient protection (i.e., "Protect your patients! Protect yourself!", "Be prepared. Get vaccinated!", "Get informed! Get vaccinated! Get protected!").

\section{Cross sectional survey}

In December 2013, we assessed the HCWs' attitudes and practices towards seasonal influenza vaccination and their opinion regarding the communication campaign. The study population included physicians, nurses, other HCWs (including students and trainees). We performed a cross-sectional survey in all intensive care units $(n=5)$ and in a random sample of $21 \%$ of the hospital noncritical inpatient medical and surgical units $(n=7 / 33)$. Overall, a total of 12 wards were included in the study.

During the day shift of selected days, we invited HCWs to fill in an anonymous, self-administered paper questionnaire, including the following information: age group $(<35,35-44,45-54, \geq 55$ years); professional category (physician, nurse, other profession); type of ward (intensive care, medical, surgical); years of service $(<5$, $5-10,>10)$; self-reported influenza immunization history (having ever received the vaccination); year of first influenza vaccination $(<2008 ; 2008-2011$; $>2011)$; adherence to annual revaccination; main reason for vaccination, missed vaccination, and missed annual revaccination; opinion regarding the communication campaign (having seen the tools, usefulness, perceived key messages).

\section{Statistical analysis}

Data were entered in a dedicated database. We performed a descriptive analysis of socio-demographic and professional characteristics, vaccination history, attitude towards and uptake of influenza vaccine, and exposure 
to the toolkit. Proportions were calculated excluding missing values. The Chi-squared or Fisher exact tests were used for univariate analyses with $p<0.05$ being considered statistically significant. The Odds Ratios (ORs) with $95 \%$ confidence intervals $(95 \% \mathrm{CI})$ were used to evaluate the association between sociodemographic and professional characteristics and having ever been vaccinated against influenza. Covariates identified as potential predictors of influenza vaccination at the univariate analysis were considered as candidates for the multivariable analysis. Logistic regression was used to assess independent predictors of self-reported influenza vaccination; the outcome of interest was having ever been vaccinated against influenza or not. The analysis was performed using the STATA software v. 12.

\section{Seasonal influenza vaccination rate}

We evaluated the annual seasonal influenza vaccination coverage rates (VC) at the hospital level, from 2009 to 2013. Annual coverage rates were estimated by using the number of seasonal influenza vaccine doses administered to HCWs as the numerator, and the number of OPBG HCWs as the denominator. Being the OPBG's HCWs' population stable over the years, we used the 2013 data as reference (1813 HCWs including 619 physicians, 1075 nurses and 119 other HCWs).

The study was approved by the Hospital Committee for Infection Control; there was no intervention on participants, and written informed consent was not required.

\section{Results}

A total of $191 \mathrm{HCW}$ participated in the survey; the response rate was $90.8 \%(109 / 120)$ for nurses and $83.7 \%$ (41/49) for physicians. Other HCWs who were on duty in the selected units in the days of the survey responded to the questionnaire (i.e. personnel dedicated to more than one unit or volunteering); due to the uncertainty of the denominator, it was not possible to calculate the response for these professional categories. Most respondents were nurses $(57 \%)$, worked in intensive care units (53.4\%), and had more than 10 years of service (43.7\%) (Table 1). Overall, 68 (35.6\%) respondents reported at least one influenza vaccination during their life; the majority of them received their first influenza vaccine between 2008 and 2011 (49/68; $72.1 \%)$. Only $6.8 \%$ of all participants (13/191), and $19.1 \%$ of participants previously vaccinated (13/68) reported to adhere to annual revaccination.

Approximately $75 \%(144 / 191)$ of participants had seen at least one promotion tool; $65.5 \%$ of the respondents who had seen the tools (93/142) found the information useful. The main perceived message for promoting vaccination was risk of influenza transmission from HCWs to patients (46\% of respondents) (Table 1). Perceived
Table 1 Characteristics of HCWs, self-reported vaccination history and opinion regarding the communication campaign; OPBG, 2013 (Number of participants: 191; number of respondents to each item are reported in brackets)

\begin{tabular}{|c|c|c|}
\hline & Number & $\%$ \\
\hline \multicolumn{3}{|l|}{ Age group, years $(n=190)$} \\
\hline$<35$ & 74 & 39.0 \\
\hline $35-44$ & 58 & 30.5 \\
\hline $45-54$ & 46 & 24.2 \\
\hline$\geq 55$ & 12 & 6.3 \\
\hline \multicolumn{3}{|l|}{ Professional category $(n=191)$} \\
\hline Nurse & 109 & 57.0 \\
\hline Physician & 41 & 21.5 \\
\hline Other HCWs & 41 & 21.5 \\
\hline \multicolumn{3}{|l|}{ Type of ward $(n=191)$} \\
\hline Intensive care & 102 & 53.4 \\
\hline Medical & 56 & 29.3 \\
\hline Surgical & 33 & 17.3 \\
\hline \multicolumn{3}{|l|}{ Years of service $(n=190)$} \\
\hline$<5$ & 50 & 26.3 \\
\hline $5-10$ & 57 & 30.0 \\
\hline$>10$ & 83 & 43.7 \\
\hline Ever vaccinated against influenza & 68 & 35.6 \\
\hline \multicolumn{3}{|l|}{ If yes, year of first influenza vaccination $(n=68)$} \\
\hline$<2008$ & 15 & 22.1 \\
\hline $2008-2011$ & 49 & 72.1 \\
\hline$>2011$ & 4 & 5.8 \\
\hline If yes, adherence to annual revaccination $(n=68)$ & 13 & 19.1 \\
\hline Exposure to at least one communication tool & 144 & 75.4 \\
\hline \multicolumn{3}{|l|}{ Usefulness of communication campaign $(n=142)$} \\
\hline Yes & 93 & 65.5 \\
\hline No & 20 & 14.1 \\
\hline Do not know & 29 & 20.4 \\
\hline \multicolumn{3}{|l|}{ Key perceived message $(n=139)$} \\
\hline Risk of influenza transmission to patients & 64 & 46.0 \\
\hline Vaccination is important/Influenza is dangerous & 42 & 30.2 \\
\hline Others & 4 & 2.9 \\
\hline Do not know & 29 & 20.9 \\
\hline
\end{tabular}

key messages did significantly vary by vaccination status; vaccinated $\mathrm{HCW}$ reported importance of vaccination and influenza risks as the main key message 2.6 times more frequently than unvaccinated colleagues $(25 / 68$, vs $17 / 122 ; p<0.001$ ) (data not shown in the table).

Patient protection was the main reason for vaccination (34.3\%); considering influenza as a mild disease was the main reason for non-vaccination (36.9\%); poor vaccine 
effectiveness was the main reported reason for missed annual revaccination $(28.8 \%)$ (Table 2 ).

At the univariate analysis, the proportion of $\mathrm{HCWs}$ who reported to be vaccinated against influenza significantly varied by age, professional category, and years of service (Table 3). Results of the multivariate analysis show that years of service and professional category were significantly and independently associated with vaccination (OR: 2.4 for $>10$ years of service, compared to $<5$ years of service; OR: 2.6 for physicians compared to nurses).

At the hospital level, the number of doses of seasonal influenza vaccine administered to $\mathrm{HCW}$ s decreased from 539 (VC: 30 \%) in 2009, to 209 (VC: $12 \%$ ) in 2010, 184 (VC: $10 \%$ ) in 2011 and 98 (VC: 5 \%) in 2012. In 2013, 253 doses were administered (VC: 14 \%) (Fig. 1).

\section{Discussion}

Influenza vaccination coverage rarely exceeded $40 \%$ in Europe between 2008 and 2011 [14], and our study confirms that satisfactory vaccination coverage rates in HCWs are hard to achieve. According to our results, only $36 \%$ of $\mathrm{HCW}$ reported to have received influenza vaccination at least once during their life. Different factors may contribute to the HCWs' decision to receive the influenza vaccine, as reported in several studies [20-23]. In a literature review [24], self-protection was reported to be the strongest motivation of HCWs, while protecting the patient was a secondary motivation in most cases. On the contrary, in our study, $34 \%$ of HCWs put patient protection before self-protection, while protecting themselves was reported as the main reason by $22 \%$ of HCWs only. Determinants of HCWs' vaccination uptake included individual factors such as knowledge, awareness of the risk of exposure to seasonal influenza in the hospital setting and responsibility towards patients regarding the risk of influenza transmission. On the other hand, misconceptions about severity of influenza, lack of knowledge on the benefits of the vaccination, lack of time, doubts about effectiveness and concern about side-effects may all play a role in refusal. Our results confirm that the professional category is a significant and independent predictor of vaccination [22]. Among different professionals, only physicians had a vaccination rate higher than $50 \%$. Similar findings were reported in a meta-analysis, showing that being a physician increased the chances of being

Table 2 Reported reasons for vaccination, missed vaccination and missed annual revaccination among HCWs; OPBG, 2013 (Number of respondents to each item are reported in brackets)

\begin{tabular}{|c|c|}
\hline Reasons for vaccination $(n=67)$ & Number among vaccinated (\%) \\
\hline Vaccination is important to protect patients & $23(34.3)$ \\
\hline The risk to contract flu in the hospital is high & $18(26.9)$ \\
\hline To avoid illness and sick-leave & $15(22.4)$ \\
\hline Vaccine is offered free of charge at OPBG & $4(6.0)$ \\
\hline I am affected with a chronic condition & $3(4.4)$ \\
\hline Influenza is a disease potentially severe & $2(3.0)$ \\
\hline Other reasons & $2(3.0)$ \\
\hline Reasons for missed vaccination $(n=122)$ & Number among not vaccinated (\%) \\
\hline Influenza is a mild disease & $45(36.9)$ \\
\hline I do not believe in vaccination practice & $24(19.7)$ \\
\hline I am afraid of adverse events & 19 (15.6) \\
\hline I am not informed enough on the benefits & $14(11.5)$ \\
\hline Vaccination was not proposed to me & $9(7.4)$ \\
\hline I forgot / I did not have time & $4(3.2)$ \\
\hline Influenza vaccine is not effective & $3(2.5)$ \\
\hline Other reasons & $4(3.2)$ \\
\hline Reasons for missed annual revaccination $(n=52)$ & Number among not revaccinated (\%) \\
\hline Vaccine was not effective & $15(28.8)$ \\
\hline Adverse events appeared & $11(21.2)$ \\
\hline Forgetfulness or lack of time & $10(19.2)$ \\
\hline Change of attitude after the pandemic $\mathrm{H} 1 \mathrm{~N} 1$ experience & $9(17.3)$ \\
\hline Vaccination was not proposed to me & $3(5.8)$ \\
\hline Other reasons & $4(7.7)$ \\
\hline
\end{tabular}


Table 3 Predictors of self-reported influenza vaccine history among HCWs; OPBG, 2013

\begin{tabular}{|c|c|c|c|c|c|c|}
\hline & \multicolumn{2}{|c|}{ Ever vaccinated } & \multicolumn{2}{|l|}{ Univariate analysis } & \multicolumn{2}{|c|}{ Multivariable logistic model } \\
\hline & Yes & No & Unadjusted OR (95\% Cl) & $p$-value & Adjusted OR (95 \% Cl) & $p$-value \\
\hline Age group (years) & & & & & NS & \\
\hline$<35$ & $20(27.0)$ & $54(73.0)$ & Ref & & & \\
\hline $35-44$ & $24(41.4)$ & $34(58.6)$ & $1.9(0.9-4.0)$ & 0.837 & & \\
\hline $45-54$ & $16(34.8)$ & $30(65.2)$ & $1.4(0.6-3.2)$ & 0.369 & & \\
\hline$\geq 55$ & $8(66.7)$ & $4(33.3)$ & $5.4(1.4-21.3)$ & 0.007 & & \\
\hline \multicolumn{7}{|c|}{ Professional category } \\
\hline Nurse & $33(30.3)$ & $76(69.7)$ & Ref & & Ref & \\
\hline Physician & $21(51.2)$ & $20(48.8)$ & $2.4(1.1-5.1)$ & 0.018 & $2.6(1.2-5.7)$ & 0.014 \\
\hline Other profession & $14(34.2)$ & $27(65.8)$ & $1.2(0.6-2.6)$ & 0.650 & $1.2(0.5-2.6)$ & 0.664 \\
\hline Type of ward & & & & & NS & \\
\hline Intensive care & $31(30.4)$ & $71(69.6)$ & Ref & & & \\
\hline Medical & $22(39.3)$ & $34(60.7)$ & $1.5(0.7-2.9)$ & 0.259 & & \\
\hline Surgical & $15(45.5)$ & $18(54.5)$ & $1.9(0.8-4.3)$ & 0.114 & & \\
\hline \multicolumn{7}{|l|}{ Years of service } \\
\hline$<5$ & $12(24.0)$ & $38(76.0)$ & Ref & & Ref & \\
\hline $5-10$ & $24(42.1)$ & $33(57.9)$ & $2.3(1.0-5.4)$ & 0.05 & 2.2 & 0.071 \\
\hline$>10$ & $32(38.5)$ & $51(61.5)$ & $1.9(0.9-4.4)$ & 0.09 & 2.4 & 0.040 \\
\hline
\end{tabular}

NS not significant

vaccinated, while being a nurse was associated with a lower vaccination rate [25].

The lower adherence to influenza vaccination reported by nurses compared to physicians is a reason of concern, as nurses have closer and more frequent contacts with patients. Thus, non vaccinated nurses may increase the risk of in-hospital influenza transmission. As reported by other authors [22], another significant determinant of vaccination was years of service, with a lower adherence in HCWs with $<5$ working years (24\%), and in those below 35 years of age (27\%), who should be properly educated regarding the risk of influenza in the healthcare setting.
The 2009 A/H1N1 pandemic influenza experience might have played a role in decreasing the compliance to vaccination during the last seasons [26]. Few HCWs adhered to annual revaccination; among those who did not adhere, $17 \%$ reported that their perception regarding the vaccination changed after the pandemic season. Moreover, hospital data show a sharp decrease in vaccination coverage from 2009 onward.

Misconceptions about influenza and influenza vaccine can be addressed by information and education [12]. To this regard, the HProImmune communication toolkit was positively evaluated by responding HCWs; however, our results show that the key messages regarding the importance

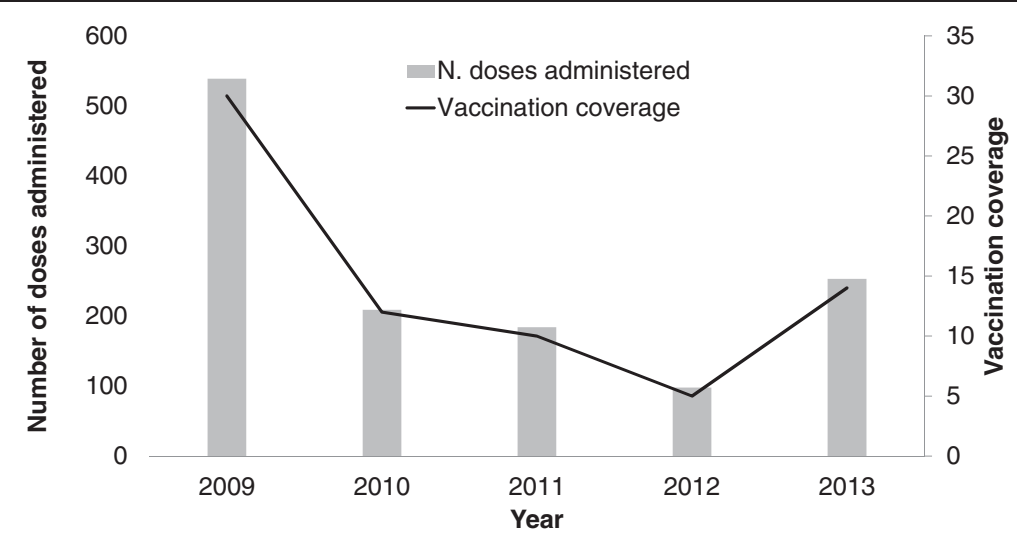

Fig. 1 Number of influenza vaccine doses and vaccination coverage by year; OPBG, 2009-2013 
of vaccination and influenza risks were perceived more strongly by those who were previously vaccinated.

The study population was represented by HCWs from all hospital Intensive Care Units and from a random sample of other in-patient wards. Participation rate among physicians and nurses was $>80 \%$, but we cannot exclude that HCWs who refused participation had different attitudes and practices towards influenza vaccination. Also, the survey did not target HCWs from outpatient clinics, who may take care of children with minor illnesses and may have different opinions regarding reasons for vaccination, such as protecting patients. Even taking into account these limitations, our results suggest that vaccine promotion should take into account specific information and communication strategies, addressing concerns of HCWs who were never vaccinated and aiming at reinforcing the value of immunization for protecting their health. Tailored educational programs for nurses should also be implemented, and influenza vaccination should be included in the initial orientation programs of all HCWs.

The benefits of HCWs' influenza vaccination have been repeatedly shown and its promotion among HCWs is recognized as a public health priority [27-29]. In OPBG, the hospital vaccination coverage rate reached a maximum of $30 \%$ in 2009, and was as low as $5 \%$ in 2012, when a precautionary recall was issued in Italy for two vaccine formulations, due to the identification of visible protein aggregates in one batch [30]. Such recall caused a shortage of influenza vaccines, as well as concerns regarding vaccine safety. In 2013, vaccination coverage reported by other Authors for Italian HCWs returned to rates observed prior to 2012 [18]. In coincidence with the implementation of the toolkit, we observed an increase of adherence to vaccination, which reached the highest coverage rate since 2009, though remaining clearly suboptimal.

\section{Conclusions}

Despite a strategy that included free vaccination offer during working hours, easy access to vaccine, and use of a standardised communication toolkit, the hospital influenza vaccination programme has been poorly effective. Interventions such as institution of declination statements, adding influenza vaccination to financial incentive systems, or vaccination as prerequisite for recruitment $[12,29,31]$ should be considered to increase influenza vaccination coverage rates among HCWs.

\section{Competing interests}

The authors declare that they have no competing interests.

\section{Authors' contributions}

VC acted as principal investigator, contributed to the study design, analyzed the data and drafted the manuscript. VA and MCR contributed to the study design and commented on the manuscript. VP contributed to data collection and interpretation. MCdA contributed to the study design, acted as study supervisor and commented on the manuscript. All authors read and approved the final manuscript.

\section{Acknowledgement}

The research has been conducted and funded in the framework of the DG SANCO Public Health Program 2008-2013, Project HProimmune, Grant Agreement N. 2010-11-02. We thank Dr. Francesco Gesualdo for language revision. We acknowledge Gaetano Ciliento and Marco Roberti who collaborated in the organization of the vaccination campaign and in the data collection during the survey. We thank all the health care workers of the Bambino Gesù Children's Hospital that participated in the survey.

\section{Author details}

'Department of Medical and Surgical Sciences, University of Foggia, Foggia, Italy. ${ }^{2}$ Medical Direction, Bambino Gesù Children's Hospital, Rome, Italy. ${ }^{3}$ Istituto Superiore di Sanità (ISS), National Centre for Epidemiology Surveillance and Health Promotion, Rome, Italy. ${ }^{4}$ Clinical Epidemiology Unit, Medical Direction, Bambino Gesù Children's Hospital, Piazza Sant'Onofrio, 4, Rome 00165, Italy.

Received: 8 March 2015 Accepted: 16 July 2015 Published online: 24 July 2015

\section{References}

1. World Health Organization. Vaccines against influenza WHO position paper - November 2012, Weekly epidemiological record. 23 November 2012, 87th. Available from: www.who.int/wer/2012/wer8747.pdf

2. European Centre for Disease Prevention and Control. Seasonal influenza vaccination in Europe -Overview of vaccination recommendations and coverage rates in the EU Member States for the 2012-13 influenza season. Stockholm: ECDC; 2015. Available from: http://ecdc.europa.eu/en/ publications/Publications/Seasonal-influenza-vaccination-Europe -2012-13.pdf.

3. Advisory Committee on Immunization Practices (ACIP). Recommended adult immunization schedule: United States, 2013. Ann Intern Med. 2013;158(3):191-9.

4. Ministry of Health. Piano Nazionale Prevenzione Vaccinale (PNPV) 2012-2014. [National Immunization Prevention Plan 2012-2014]. Gazzetta Ufficiale. n. 60. 12 Mar 2012. Italian. Available from: http://www.salute.gov.it/ imgs/C_17_pubblicazioni_1721_allegato.pdf

5. Vanhems P, Voirin N, Roche S, Escuret V, Regis C, Gorain C, et al. Risk of influenza-like illness in an acute health care setting during community influenza epidemics in 2004-2005, 2005-2006, and 2006-2007: a prospective study. Arch Intern Med. 2011;171(2):151-7.

6. Chironna M, Tafuri S, Santoro N, Prato R, Quarto M, Germinario CA. A nosocomial outbreak of 2009 pandemic influenza $A(\mathrm{H} 1 \mathrm{~N} 1)$ in a paediatric oncology ward in Italy, October-November 2009. Euro Surveill. 2010;7:15(1).

7. Mejia C, Silvestre M, Cazali I, Garcia J, Sanchez R, Garcia L, et al. Large epidemiological influenza a outbreak in a teaching hospital from Guatemala city. ISRN AIDS. 2012;2012:638042

8. Regis C, Voirin N, Escuret V, Kim BA, Robert O, Lina B, et al. Five years of hospital based surveillance of influenza-like illness and influenza in a short-stay geriatric unit. BMC Res Notes. 2014;7(1):99.

9. Yassi A1, McGill M, Holton D, Nicolle L. Morbidity, cost and role of health care worker transmission in an influenza outbreak in a tertiary care hospital. Can J Infect Dis. 1993:4(1):52-6.

10. Mota NV, Lobo RD, Toscano CM, Pedroso De Lima AC, Souza Dias MB, Komagata $\mathrm{H}$, et al. Cost-effectiveness of sick leave policies for health care workers with influenza-like illness, Brazil, 2009. Emerg Infect Dis. 2011;17(8):1421-9. doi:10.3201/eid1708.101546.

11. Ahmed F, Lindley MC, Allred N, Weinbaum CM, Grohskopf L. Effect of influenza vaccination of healthcare personnel on morbidity and mortality among patients: systematic review and grading of evidence. Clin Infect Dis. 2014:58(1):50-7.

12. Ajenjo MC, Woeltje KF, Babcock HM, Gemeinhart N, Jones M, Fraser VJ. Influenza vaccination among healthcare workers: ten-year experience of a large healthcare organization. Infect Control Hosp Epidemiol. 2010;31(3):233-40. 
13. Influenza vaccination coverage among health-care personnel - United States. 2010-11 influenza season. MMWR Morb Mortal Wkly Rep. 2011;60(32):1073-7.

14. Mereckiene J, Cotter S, Nicoll A, Lopalco P, Noori T, Weber JT, D'Ancona F, Lévy-Bruhl D, Dematte L, Giambi C, Valentiner-Branth P, Stankiewicz I, Appelgren E, O'Flanagan D, the VENICE project gatekeepers group. Seasonal influenza immunisation in Europe. Overview of recommendations and vaccination coverage for three seasons: pre-pandemic (2008/09), pandemic (2009/10) and post-pandemic (2010/11). Euro Surveill.

2014;19(16):pii = 20780. Available online: http://www.eurosurveillance.org/ ViewArticle.aspx?Articleld $=20780$

15. Esposito S, Bosis S, Pelucchi C, Tremolati E, Sabatini C, Semino M, et al. Influenza vaccination among healthcare workers in a multidisciplinary University hospital in Italy. BMC Public Health. 2008;8:422.

16. Blank PR, Schwenkglenks M, Szucs TD. Vaccination coverage rates in eleven European countries during two consecutive influenza seasons. J Infect. 2009:58(6):446-58.

17. Rizzo C, Bella A, Declich S, Rota MC, e il gruppo di lavoro influenza pandemica. Sorveglianza epidemiologica integrata della pandemia influenzale da virus A/H1N1v nella stagione 2009-2010. Roma: Istituto Superiore di Sanità; 2010. Rapporti ISTISAN 10/46.

18. Alicino C, ludici R, Barberis I, Paganino C, Cacciani R, Zacconi M, et al. Influenza vaccination among healthcare workers in Italy. Hum Vaccin Immunother. 2015;11(1):95-100.

19. HProlmmune. Promotion of Immunization for Health Professionals in Europe. Available from: http://www.hproimmune.eu/

20. Van den Dool C, Van Strien AM, den Akker IL, Bonten MJ, Sanders EA, Hak E. Attitude of Dutch hospital personnel towards influenza vaccination. Vaccine. 2008;26(10):1297-302.

21. Mytton OT, O'Moore EM, Sparkes T, Baxi R, Abid M. Knowledge, attitudes and beliefs of health care workers towards influenza vaccination. Occup Med (Lond). 2013;63(3):189-95.

22. Hollmeyer $\mathrm{HG}$, Hayden F, Poland G, Buchholz U. Influenza vaccination of health care workers in hospitals-a review of studies on attitudes and predictors. Vaccine. 2009;27(30):3935-44.

23. Barbadoro P, Marigliano A, Di Tondo E, Chiatti C, Di Stanislao F, D'Errico MM, et al. Determinants of influenza vaccination uptake among Italian healthcare workers. Hum Vaccin Immunother. 2013;9(4):911-6.

24. Hofmann F, Ferracin C, Marsh G, Dumas R. Influenza vaccination of healthcare workers: a literature review of attitudes and beliefs. Infection. 2006;34(3):142

25. Riphagen-Dalhuisen J, Gefenaite G, Hak E. Predictors of seasonal influenza vaccination among healthcare workers in hospitals: a descriptive meta-analysis. Occup Environ Med. 2012;69(4):230-5.

26. Brandt C, Rabenau HF, Bornmann S, Gottschalk R, Wicker S. The impact of the 2009 influenza $A(\mathrm{H} 1 \mathrm{~N} 1)$ pandemic on attitudes of healthcare workers toward seasonal influenza vaccination 2010/11. Euro Surveill. 2011;28:16(17).

27. Potter J, Stott DJ, Roberts MA, Elder AG, O'Donnell B, Knight PV, et al. Influenza vaccination of health care workers in long-term-care hospitals reduces the mortality of elderly patients. J Infect Dis. 1997;175(1):1-6.

28. WHO. Resolution WHA 56.19. Prevention and control of influenza pandemics and annual epidemics. Geneva: Fifty-sixth World Health Assembly; 2003. p. 19-5

29. CDC. Immunization of health-care personnel: recommendations of the Advisory Committee on Immunization Practices (ACIP). MMWR Recomm Rep. 2011;60(RR-7):1-45.

30. Agenzia Italiana del Farmaco. Information AIFA/PQ/P1176884, 24 October 2012. Available from: http://www.agenziafarmaco.gov.it/sites/default/files/ divieto_di_utilizzo_vaccini_influenzali.pdf

31. Galanakis E, Jansen A, Lopalco PL, Giesecke J. Ethics of mandatory vaccination for healthcare workers. Euro Surveill. 2013; 18 (45):pii = 20627. Available from: http://www.eurosurveillance.org/ViewArticle.aspx? Articleld $=20627$

\section{Submit your next manuscript to BioMed Central and take full advantage of:}

- Convenient online submission

- Thorough peer review

- No space constraints or color figure charges

- Immediate publication on acceptance

- Inclusion in PubMed, CAS, Scopus and Google Scholar

- Research which is freely available for redistribution

Submit your manuscript at www.biomedcentral.com/submit 